\title{
CONTINGENCY PLANNING FOR THE MICROWAVE ANISOTROPY PROBE MISSION
}

\author{
Michael A. Mesarch" \\ National Aeronautics and Space Administration \\ Goddard Space Flight Center. Code 572 \\ Greenbelt, MD 20771 \\ David Rohrbaugh ${ }^{*} \&$ Conrad Schiff* \\ a.i. solutions, Inc. \\ 10001 Derekwood Lane, Suite 215 \\ Lanham. MD 20706
}

\begin{abstract}
The Microwave Anisotropy Probe (MAP) utilized a phasing loop/lunar encounter strategy to achieve a small amplitude Lissajous orbit about the SunEarth/Moon L2 libration point. The use of phasing loops was key in minimizing MAP's overall $\Delta V$ needs while also providing ample opportunities for contingency resolution. This paper will discuss the different contingencies and responses studied for MAP. These contingencies included accommodating excessive launch vehicle errors (beyond $3 \sigma$ ), splitting perigee maneuvers to achieve ground station coverage through the Deep Space Network (DSN), delaying the start of a perigee maneuver, aborting a perigee maneuver in the middle of execution, missing a perigee maneuver altogether, and missing the lunar encounter (crucial to achieving the final Lissajous orbit). It is determined that using a phasing loop approach permits many opportunities to correct for a majority of these contingencies.
\end{abstract}

\section{Introduction}

The Microwave Anisotropy Probe, launched on June 30,2001 , was the second spacecraft launched as part of NASA's Medium Explorer (MIDEX) program. From its Lissajous orbit about the Sun-Earth/Moon L2 libration point, MAP will take measurements of the cosmic microwave background energy left over from the Big Bang, serving as a follow-up mission to the highly successful Cosmic Background Explorer (COBE) mission. In order to reach it's orbit about L2, MAP employed an elliptical phasing loop strategy to accurately target a lunar gravity assist, necessary to provide MAP with sufficient energy to reach L2. The lunar encounter targeting was performed using maneuvers at the phasing loop perigees as control variables with encounter parameters at the Moon (BPlane') as goals. Figure 1 shows a schematic of a 3 loop scenario to meet the Moon - both 3-loop and 5loop scenarios were deemed viable for $\mathrm{MAP}^{2}$. After transfer trajectory insertion (TTI) from the launch vehicle, opportunities existed at each of the perigees (P1, P2, etc.) to adjust the lunar encounter at periselene (closest approach to the Moon). Maneuvers at apogee (A1, A2, etc.) were only used as a control to keep the perigee height from violating the mission constraint of $500 \mathrm{~km}$. A final perigee correction maneuver ( $\mathrm{PfCM}$ ) was needed to correct for execution errors from the final perigee maneuver while a mid-course correction (MCC) maneuver was used to fine-tune the trajectory following the gravity assist. MAP maintains its Lissajous orbit about L2 through periodic, small stationkeeping maneuvers - approximately $1 \mathrm{~m} / \mathrm{s}$, four times per year. MAP's Lissajous orbit at L2 was designed to stay within a $0.5^{\circ}$ by $10.5^{\circ}$ corridor. The angles described here are defined as the angle between the Earth-MAP vector and the Earth-L2 vector (essentially, the Sun-Earth line). The lower limit rules out the appearance of Earth eclipses while the higher limit is a communications constraint.

Due to the mass growth of the spacecraft during its development phase, MAP was $\triangle V$-limited by the capacity of its full propellant tank. MAP carries a monopropellant hydrazine propulsion system (blowdown) containing a beginning-of-life propellant load of $72.5 \mathrm{~kg}$ and eight $1-\mathrm{lb}$ thrusters (Figure 2). To further complicate matters, thruster cant angles, finite burn losses, and ACS dutycycling required portions of the available $\triangle \mathrm{V}$ necessary to design a trajectory that met all of MAP's mission constraints. Due to these limitations, $70 \mathrm{~m} / \mathrm{s}$ of $\Delta \mathrm{V}$ (impulsive) was allocated for execution of the phasing loop maneuvers at perigee and apogee. An additional $15 \mathrm{~m} / \mathrm{s}$ was allocated to the PfCM and $10 \mathrm{~m} / \mathrm{s}$ was allocated to the MCC maneuver. With these $\triangle \mathrm{V}$ constraints, the MAP trajectory designers were able to determine roughly 10 days per month when MAP could launch and meet all trajectory constraints - including $\pm 3 \sigma$ launch vehicle errors. These launch opportunities occurred during two blocks of days: 6 days utilizing 3 phasing loops and 4 days utilizing 5 phasing loops. These 3- and 5-loop launch

\footnotetext{
* Flight Dynamics Engineer, Guidance, Navigation, and Control Center, Senior Member AIAA

+ Systems Engineer, a i. Solutions, Inc.

* Chief Scientist, a.i. Solutions, Inc.
} 
blocks were usually separated by 10 days $^{3}$. The total $\Delta V$ capability of the MAP propulsion system is 151 $\mathrm{m} / \mathrm{s}$, assuming $-3 \sigma$ performance by the thrusters. Since the trajectories for each candidate launch date were different, the excess available $\Delta V$ (up to the $70 \mathrm{~m} / \mathrm{s}$ limit) varied as well. This excess $\Delta \mathrm{V}$ would be available for contingency resolution. Looking at all candidate launch dates revealed an average of $41 \mathrm{~m} / \mathrm{s}$ of $\triangle \mathrm{V}$ available for contingency resolution. Using MAP's actual launch date of June 30, 2001 as an example, a $\Delta V$ of $47 \mathrm{~m} / \mathrm{s}$ was available for contingency resolution. It is important to note that the $151 \mathrm{~m} / \mathrm{s}$ capability is a worst-case number. In all likelihood, the propulsion system would perform much better than the $-3 \sigma$ prediction.

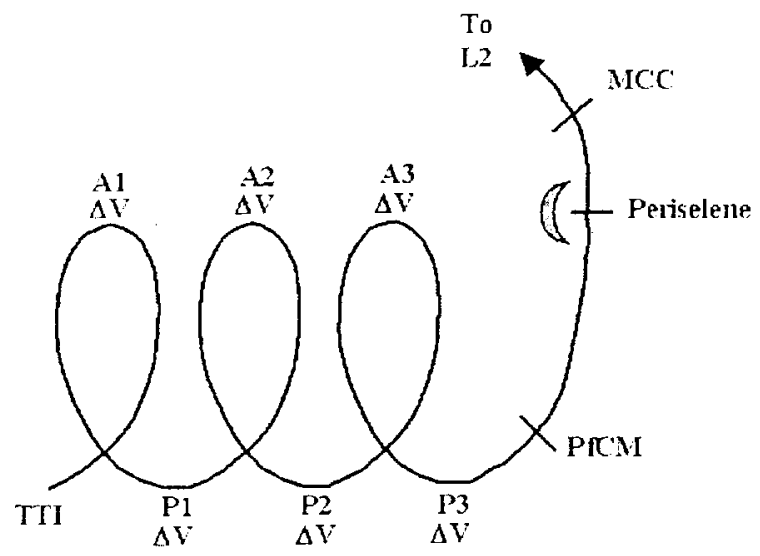

Figure 1: MAP 3-Loop Representation
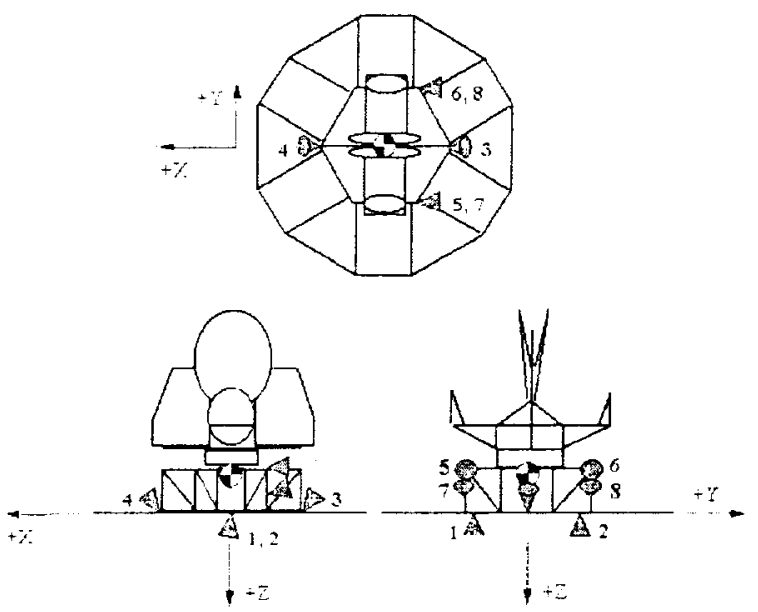

Figure 2: MAP Thruster Layout

\section{Contingency Analysis}

While a great amount of effort went into determining MAP's available launch opportunities, a parallel effort was underway to understand what happened if something went wrong after launch. During the year prior to launch, the MAP trajectory team worked to identify the potential contingency scenarios that were likely to occur. After analyzing these scenarios, responses were devised for recovery from these problems, or, in the event that recovery was not possible, ways to mitigate a contingency were conceived. The contingencies related to the trajectory design that were considered included:

(5) Recovery from launch vehicle errors in excess of $3 \sigma$

$\Leftrightarrow$ Splitting a perigee maneuver to achieve ground station coverage

5 Delaying the start of a maneuver

7) Aborting a maneuver prior to complete execution

$\Rightarrow$ Missing a maneuver

(7) Missing the lunar encounter

While the excessive launch vehicle error and missing the lunar encounter scenarios were preformed before launch, the remaining items were analyzed during the preparation for each maneuver. As a particular phasing loop maneuver planning was being performed, a separate contingency study was being performed to determine the effects of delaying, aborting, or missing the maneuver. The results of these studies were presented during the maneuver command authorization meetings (CAM). The CAM was the final meeting where all maneuver activities were discussed before the command sequence was uploaded to the spacecraft and the maneuver was allowed to proceed.

The following section discusses the analysis of these contingency scenarios and the resolution needed to correct for each situation. A majority of the contingency analyses were performed relative to the June $30^{\text {th }}$ launch date and any exceptions are noted. In these exceptions, application of the results to the actual MAP launch date will be discussed.

\section{Excessive Launch Vehicle Errors}

One of the first contingencies examined concerned the possibility of excessive launch vehicle errors - beyond the traditional $\pm 3 \sigma$. All MAP trajectories were designed to be able to compensate for $\pm 3 \sigma$ launch vehicle errors through the re-distribution of the perigee $\Delta V$ maneuvers. The Boeing launch vehicle analysts assigned to MAP a $3 \sigma$ launch dispersion of $11.6 \mathrm{~m} / \mathrm{s}$. This dispersion is on the $\Delta V$ from third stage of the Delta-II 7425-10, a Star-48B solid rocket motor produced by the Thiokol Corporation. A pointing dispersion of $2^{\circ}$ was also provided by Boeing but was not considered for this study after observing that the energy dispersion was the dominant factor ${ }^{j}$. To analyze 
this contingency, launch vehicle errors from $4 \sigma$ up to 50 were examined by including the appropriate $\Delta \mathrm{V}$ dispersion. Using STK/Astrogator (the mission design tool used for MAP, developed by Analytical Graphics, Inc.), a differential correction targeting scheme was used to re-distribute the phasing loop $\Delta V$ until appropriate B-plane parameters were met at periselene ${ }^{6}$. Once a suitable lunar encounter was found, the Lissajous orbit was examined to determine if the mission orbit satisfied the $0.5^{\circ} \times 10.5^{\circ}$ size constraint. Other trajectory constraints included:

$\checkmark$ Minimum phasing loop perigee height $=500$ $\mathrm{km}$ (for attitude stability)

$\checkmark$ Maximum final perigee maneuver $=30 \mathrm{~m} / \mathrm{s}$ (consistent with PfCM allocation of $15 \mathrm{~m} / \mathrm{s}$ )

$\checkmark$ No shadows (Earth or lunar) for the two year mission at $\mathrm{L} 2$

In Figure 3 , the $\Delta \mathrm{V}$ penalty is shown as the launch vehicle error grows from $\pm 3 \sigma$ up to $\pm 5 \sigma$ for the June 30,2001 launch date. In all cases, the trajectory can meet all mission constraints within the contingency margin of $47 \mathrm{~m} / \mathrm{s}$. It is important to note that the $-\sigma$ cases are all subjected to unfavorable lunar perturbations that force the altitude of first perigee (P1) below $500 \mathrm{~km}$, necessitating an apogee maneuver at first apogee. Furthermore, all of the $-\sigma$ cases have very large Pl maneuvers (greater than $40 \mathrm{~m} / \mathrm{s}$ ). These maneuvers would incur a very large finite maneuver loss (greater than $35 \%$ ) due to the high eccentricity of the phasing loop? On a beneficial side, the final perigee maneuver at $\mathrm{P} 3$ is very small (less than $2 \mathrm{~m} / \mathrm{s}$ ) for all the $-\sigma$ cases. A small P3 maneuver ensures that the PfCM stays well below the $15 \mathrm{~m}$ /s allocation.

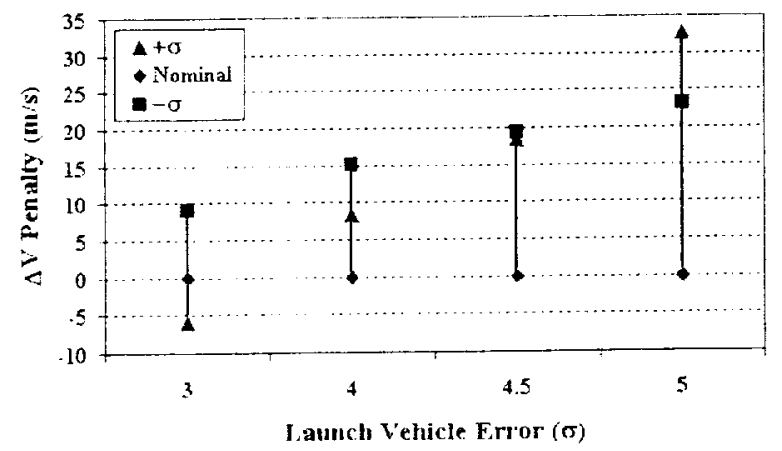

Figure 3: Penalty for Launch Vehicle Errors

The $+\sigma$ cases exhibit a much different trend. The redistribution of the perigee maneuver $\Delta V$ forces a large retrograde $\mathrm{P} 1$ in addition to a large $\mathrm{P} 3$ maneuver. In fact, for the $+5 \sigma$ case, the P3 maneuver was fixed at the
$30 \mathrm{~m} / \mathrm{s}$ requirement and extra $\Delta \mathrm{V}$ was required at $\mathrm{P} 2$ in order to meet the encounter goals. Fortunately, the probability of an excessive $+\sigma$ performance is unlikely, due to the realities of launch vehicle performance. In the end, MAP was launched into its phasing loops with nearly nominal performance (within $0.25 \sigma$ ) and no contingency $\Delta \mathrm{V}$ was required.

\section{Splitting Perigee Maneuvers}

Due to MAP's low perigee altitudes in the phasing loops, the probability of passing over a Deep Space Network (DSN) ground station during perigee maneuvers was not very high. It was for this reason that the MAP project decided to require telemetry and comnand capability through the Tracking and Data Relay Satellite (TDRS) System. However, if a contingency arose whereby a link through TDRS was not possible, zero telemetry would be available for MAP's perigee passes. Therefore, the effect of splitting the perigee maneuver into two burns, one on either side of perigee, to obtain ground station coverage was studied $^{8}$. This analysis was only performed on maneuvers at first perigee (P1). It was decided that, due its criticality, no attempt would be made to alter the final perigee maneuver and it would be performed without station coverage, if necessary

Four separate launch days were analyzed: 11/08/2000, $12 / 07 / 2000,02 / 21 / 2000$, and $04 / 18 / 2000$ - these were early MAP launch opportunities. The first two were 3loop cases while the latter two were 5-loop cases. For this analysis, the maneuver at P1 for each case was divided into two separate maneuvers. The first maneuver was placed during a station contact prior to $\mathrm{Pl}$ and the second part of the maneuver was positioned during a station contact after $\mathrm{P} 1$. It is reasonable to assume that there would be a penalty to implement this scenario because these split maneuver would be performed off of the optimal perigee location. The goal was to determine the $\Delta \mathrm{V}$ penalty of splitting up the $\mathrm{Pl}$ burn in various ways that would allow the analysts to target back to the same lunar encounter. Station contact reports were generated in STK for each of the four cases in order to choose an appropriate time for executing the split maneuvers. Figure 4 shows a sample station coverage scenario around perigee. In this example, station DS46 (Canberra, Australia) has coverage prior to perigee while stations DS16 (Goldstone, USA) and DS66 (Madrid, Spain) have coverage after perigee. 


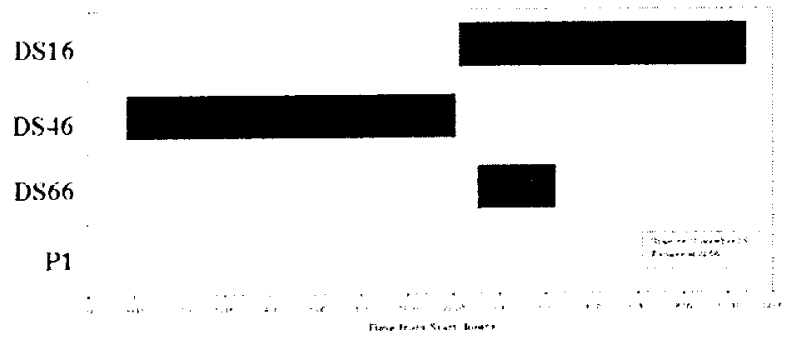

Figure 4: DSN Coverage Around First Perigee

The split perigee maneuvers were performed during station contacts approximately two hours prior to and two hours after perigee. The maneuvers were not allowed to occur too close to the beginning or to the end of a pass because of operational issues. This buffer would allow time for a post-maneuver performance assessment of the pre-perigee maneuver prior to loss of signal. Likewise, the buffer would provide time for a pre-maneuver checkout of the spacecraft after acquisition of signal after perigee. A buffer time of two hours was chosen for this study.

Three different re-targeting strategies were used for each of the four cases. Strategy 1 involved targeting only the split P1 maneuvers, keeping the remaining maneuvers fixed. Strategy 2 fixed each of the split P1 maneuvers to half the original P1 value and re-targeted the remaining maneuvers. Strategy 3 retargeted all of the maneuvers. The procedure for each of these strategies was identical. The P1 burn was varied as per the different strategies. Next, B-Plane targeting was used to get "close" to the nominal Lissajous orbit. Once this targeting was successfully performed, a second targeting scheme was performed using the right ascension and declination of the outgoing asymptote from the lunar encounter along with the orbit energy at a point near MCC (typically 7 days after the lunar encounter $)^{9}$. This procedure allowed for targeting back to the nominal Lissajous in most of cases. Convergence was not possible on the nominal Lissajous orbit using Strategy 2 for either of the 3-loop cases, but was possible using Strategy 3. In the 5-loop cases the nominal Lissajous orbit was attainable using all three strategies, although for the February $21 \mathrm{st}$ case, the phasing loop $\Delta \mathrm{V}$ budget of $70 \mathrm{~m} / \mathrm{s}$ was exceeded using Strategy 1.

In every case, the $\Delta \mathrm{V}$ penalty for splitting the first perigee burn to obtain ground station contact is between $8 \mathrm{~m} / \mathrm{s}$ and $17 \mathrm{~m} / \mathrm{s}$, depending on the strategy. Note that this penalty is a "best" case in that it is the minimum $\Delta \mathrm{V}$ penalty of the three strategies used. It appeared that Strategy 2 or Strategy 3 provided the smallest penalty. Table 1 below shows the results of the $11 / 08 / 2000$ case. This case is shown in detail due to its similarity to the actual June 30, 2001 launch date where the $P ! \Delta V$ was $21.5 \mathrm{~m} / \mathrm{s}$ and the $\mathrm{P} 3 \Delta \mathrm{V}$ was $8.7 \mathrm{~m} / \mathrm{s}$ )

\section{Table 1: Split Perigee Penalty for 11/08/2000} Launch Case $(\Delta V$ in $\mathrm{m} / \mathrm{s})$

\begin{tabular}{|c|c|c|c|c|}
\hline & & Strategy 1 & Strategy 2 & Strateg: 3 \\
\hline Maneuver & Nom. & $\begin{array}{c}\text { Vary Split P1, } \\
\text { Hold P3 }\end{array}$ & $\begin{array}{c}\text { Hold P1, } \\
\operatorname{vary} \mathrm{P} 2 / \mathrm{P} 3\end{array}$ & Vary all 4 \\
\hline Pla & 21.5 & $21.7^{*}$ & 11.0 & $3.0^{*}$ \\
\hline Plb & $N / A$ & $25.9^{*}$ & 11.0 & $11.3^{*}$ \\
\hline $\mathrm{P} 2$ & 0.0 & 0.0 & $20.3^{*}$ & $25.9^{*}$ \\
\hline $\mathrm{P} 3$ & 10.8 & 10.8 & $1.0^{*}$ & $-0.9^{*}$ \\
\hline Total & 32.3 & 58.4 & 43.3 & 41.1 \\
\hline Penalty & 0.0 & 26.1 & 11.0 & 8.8 \\
\hline
\end{tabular}

For this date, Strategy 3 provided the smallest penalty $(8.8 \mathrm{~m} / \mathrm{s})$ and most of the phasing loop $\Delta \mathrm{V}$ has been shifted to the P2 maneuver. This attribute was also exhibited in the other 3-loop case (12/07/2000). The most fuel-efficient strategy for the 5-loop cases was Strategy 2, dividing P1 in half and allowing Astrogator to re-target the lunar encounter using maneuvers at P2 and P5. In general, Strategy 1, allowing Astrogator to target the first perigee maneuver segments while keeping the remaining burns constant, was the least efficient method.

From this study, the penalty incurred by splitting the first perigee maneuver to obtain DSN coverage was within the contingency budget. However, a lot of planning would have been required to put this plan in place, necessitating knowledge of a TDRS outage far in advance of the maneuver. A re-plan to accommodate a last-second outage of TDRS would not have been possible. Therefore, it was determined that MAP would execute it's first perigee maneuver "in the blind" in the event of an unanticipated TDRS outage.

\section{Delaving a Maneuver}

In the event of a spacecraft system failure (e.g. processor re-boot, delay in getting commands to spacecraft, etc.), it may have been necessary to delay a perigee maneuver from its optimal starting time (roughly centered about perigee). The following results are actual flight analysis performed in preparation for MAP's critical phasing loop maneuvers (P1, P2, P3, and PfCM) after its successful launch on June 30, 2001.

\section{PI Maneuver}

The nominal plan for the June 30 launch date included a $\mathrm{P} 1 \mathrm{maneuver}$ of $21.5 \mathrm{~m} / \mathrm{s}$ and a $\mathrm{P} 3$ maneuver of $8.7 \mathrm{~m} / \mathrm{s}$. In the event that the P1 maneuver was to be delayed, a $\Delta V$ penalty would be incurred due to the inefficiency of maneuvering off of perigee. In order to recover from this contingency, a maneuver would be needed at P2 to help compensate for the energy loss at P1. Furthermore, 
the P3 maneuver would be adjusted from its original value. Astrogator was used to parameterize the $\Delta \mathrm{V}$ penalty as a function of the Pl maneuver delay. Figure 5 shows the penalty incurred from delaying the $\mathrm{Pl}$ maneuver. As $\mathrm{Pl}$ is delayed, the $\mathrm{P} 2$ maneuver rises in magnitude while the P3 maneuver decreases, becoming a retrograde maneuver after a delay of 90 minutes. The $\Delta V$ penalty rises to over $12 \mathrm{~m} / \mathrm{s}$ after a delay of two hours. It is important to note that after a delay of 105 minutes, the penalty for delaying the maneuver further becomes larger than skipping the maneuver altogether (see below - Missing a Maneuver). This information was relayed to the MAP System Engineer as a potential contingency decision point.

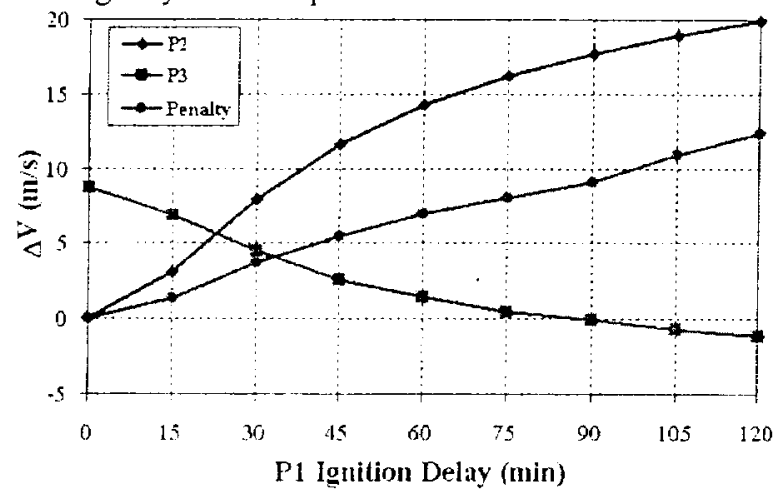

Figure 5: Penalty for Delaying P1 Maneuver

\section{P2 Maneuver}

After calibration of the actual P1 maneuver, it was determined that a small maneuver of $2.5 \mathrm{~m} / \mathrm{s}$ was needed to correct back to the nominal trajectory. With this additional maneuver at P2, the P3 maneuver shrank in size from $8.7 \mathrm{~m} / \mathrm{s}$ to $7.4 \mathrm{~m} / \mathrm{s}$. In a fashion similar to the P1 delay analysis, a delay was applied to the P2 maneuver and the P3 maneuver was re-targeted by using the start time and duration of the maneuver as control variables. Again, the constraints were the lunar encounter parameters (B-plane) at periselene. While the $\Delta V$ penalty incurred from delaying $P 2$ was small (less than $0.5 \mathrm{~m} / \mathrm{s}$ ), the need to adjust the start time of the P3 maneuver forced the Sun out of the digital sun sensor (DSS) field of view after a P2 delay of only 5 minutes. MAP requires the Sun to remain in the DSS field of view for all maneuvers ${ }^{10}$. Deeming this method unacceptable, a second strategy was used. A new attempt was made to add a control variable of adjusting the delayed P2 maneuver. This scheme maintains the square ( 2 controls $/ 2$ constraints) targeting scenario used previously. The results of this strategy are shown in Figure 6. Essentially, as the P2 maneuver is delayed, the size of the maneuver is adjusted in duration (i.e. $\Delta V)$ and hence, the P3 maneuver is adjusted accordingly. For example, if there were an 80 minute delay in the P2 maneuver, the magnitude of P2 maneuver doubles while the P3 maneuver is reduced by $18 \mathrm{~cm} / \mathrm{s}$. The end result is an overall penalty of $2.2 \mathrm{~m} / \mathrm{s}$. These small changes in the P2 maneuver maintained the geometry necessary for DSS coverage during the P3 maneuver while being able to achieve the desired lunar encounter parameters at periselene. In order to be prepared for this scenario, sufficient attitude pointing data (in the form of a quaternion history file) was uploaded to the spacecraft prior to the maneuver.

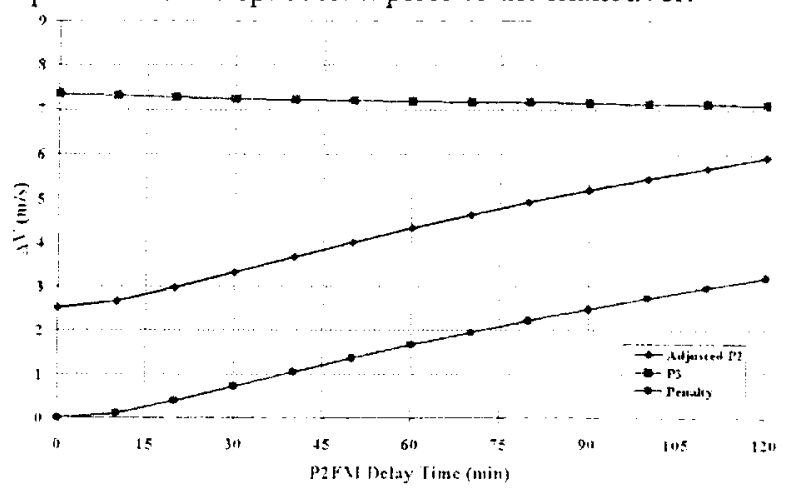

Figure 6: Penalty for P2 Maneuver Delay/Restart

\section{P3 Manetuer}

The P2 maneuver was executed near a nominal level and the $P 3$ maneuver remained at $7.4 \mathrm{~m} / \mathrm{s}$ in size. The P3 maneuver is the last opportunity to optimally add energy to the trajectory. Similar to the P2 delay analysis, the P3 maneuver duration was adjusted as a function of the delay time. This approach suffered from the lack of control variables (only one), and the $B \bullet R$ at periselene began to differ from the desired value. Figure 7 shows the penalty incurred from delaying/adjusting the P3 maneuver. Despite the small penalty, the change in $B \bullet R$ at periselene drifted enough to allow a lunar shadow (4\% depth) to appear in the cruise phase after only a 12 minute delay. Careful targeting using a maneuver at PfCM, 18 hours after P3, or at MCC would help to remove the cruise shadow.

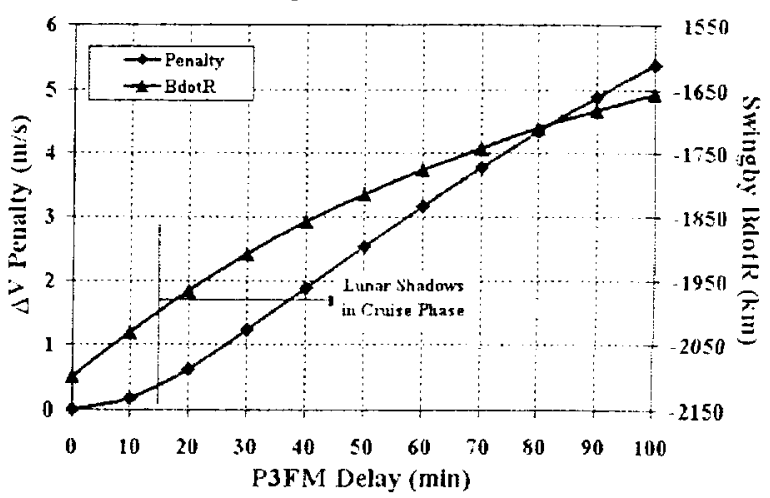

Figure 7: Penalty for Delaying P3 Maneuver 


\section{PfCM}

The PfCM is a maneuver to correct for any errors incurred from the final perigee maneuver, in this case, $\mathrm{P3}$. Also, $\mathrm{PfCM}$ is the last opportunity to correct the trajectory prior to the lunar encounter. Fortunately, P3 execution went well and the PfCM was estimated to be $0.31 \mathrm{~m} / \mathrm{s}$ (i.e. $31 \mathrm{~cm} / \mathrm{s}$ ). Some analysis was performed to estimate the penalty for delaying the PfCM maneuver. Fortunately, this maneuver was not very sensitive to maneuver delays as a twelve hour delay in the ignition time evolved into a mere $17 \mathrm{~cm} / \mathrm{s}$ penalty.

\section{Aborting a Maneuver}

If for some reason a maneuver had to be aborted in mid-execution, the remaining maneuvers could be used to compensate for the $\Delta \mathrm{V}$ deficit. Again, this fact shows the flexibility of the phasing loop strategy. A second contingency response where the aborted maneuver was restarted after some delay was also examined in some cases. The re-start strategy would only be implemented if the reason for the abort was solved quickly, if it was well understood why it had happened, and if the same problem would not affect the subsequent restart maneuver.

\section{Pl Maneuver}

As part of the contingency planning for the $\mathrm{Pl}$ maneuver, the effects of a partially completed (aborted) maneuver were examined. As in the delayed maneuver case, the response scenario was to compensate for this contingency by re-targeting the downstream maneuvers, in this case P2 and P3. The results of this parameterization are shown in Figure 8. There is no penalty, or sometimes even a little $\Delta V$ "rebate", as long as at least $50 \%$ of the P1 maneuver was completed. The penalty begins to increase (up to $10 \mathrm{~m} / \mathrm{s}$ ) at any completion percentage below $50 \%$.

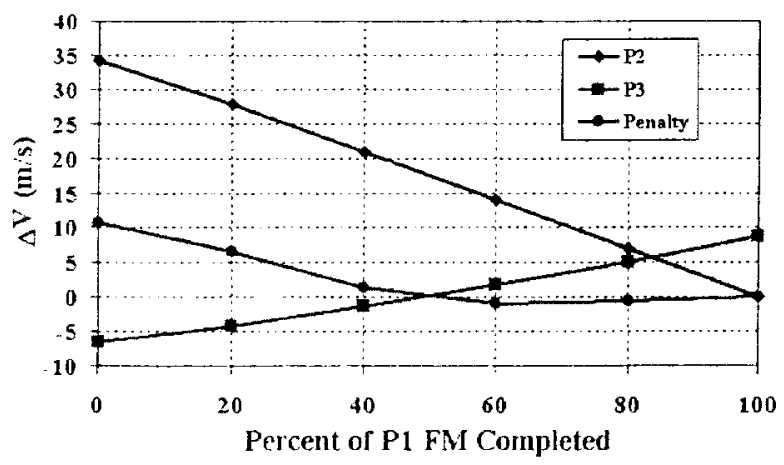

Figure 8: Penalty for Aborting P1 Maneuver

In Figure 9, the effects of possibly re-starting an aborted Pl maneuver are shown. This strategy was parameterized with respect to percent completed and the amount of time before the remainder of the maneuver was performed. For example, the penalty for completing $60 \%$ of $\mathrm{Pl}$, waiting one hour, and completing the remaining $40 \%$ of $\mathrm{P} 1$ was $2.7 \mathrm{~m} / \mathrm{s}$. As stated above though, as long as $50 \%$ of the P1 maneuver was completed, there was no penalty. The $20 \%$ and $40 \%$ abort/re-start curves show a $\Delta \mathrm{V}$ penalty of less than $8 \mathrm{~m} / \mathrm{s}$ provided the remainder of the maneuver can be initiated in two hours or less.

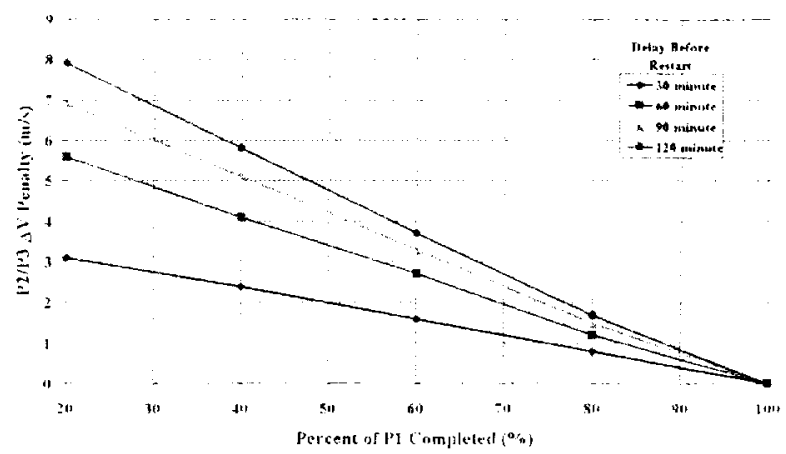

Figure 9: Penalty for P1 Maneuver Abort/Restart

\section{P2 Maneuver}

As in the P1 abort case, the P2 analysis was parameterized with the percent of the $\mathrm{P} 2$ maneuver completed and the delay before a re-start could be attempted. In this case, the second portion of P2 was adjusted in order to minimize the $\Delta \mathrm{V}$ penalty. In all of these cases, the second portion of the P2 maneuver and the P3 maneuver were adjusted in order to meet lunar encounter constraints at periselene. This correction scheme allowed the analysts to target back to the nominal trajectory while meeting all mission constraints. As can be seen from the results (Figure 10), a small penalty (less than $3 \mathrm{~m} / \mathrm{s}$ ) in incurred from aborting and restarting a maneuver. Careful examination of this plot show that the extreme left of this curve (i.e. $0 \%$ completed) matches the P2 Delay curve from Figure 6.

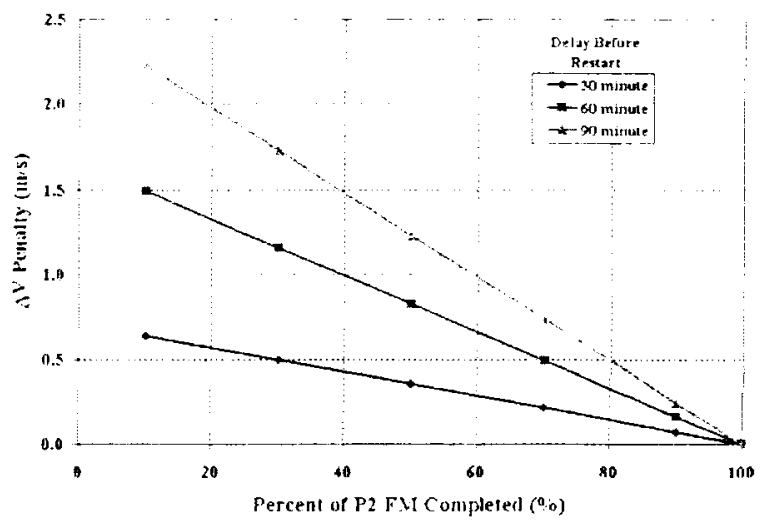

Figure 10: Penalty for Aborting | Restarting P2 Maneuver 


\section{P3 Maneuver}

After successful completion of both the P1 and P2 maneuvers, only $7.4 \mathrm{~m} / \mathrm{s}$ were needed at the $\mathrm{P} 3$ maneuver. Maneuver abort analysis was performed using two response strategies. The first strategy was to correct for the abort by adding additional $\triangle \mathrm{V}$ at PfCM, 18 hours after the P3 maneuver. This method required the targeting of both the tangential and normal components of the PfCM at control in order to meet the lunar encounter parameters at periselene. The addition of the normal component requires MAP to yaw out of the orbit plane by less than $9^{\circ}$. In this strategy, the prelaunch allocation of $15 \mathrm{~m} / \mathrm{s}$ for the PfCM was exceeded if less than $57 \%$ of the P3 maneuver was completed (Figure 11). A second response was analyzed where a P3 re-start maneuver was performed after some time delay, and the re-start maneuver magnitude was used in a single degree of freedom targeting scenario to achieve a satisfactory lunar encounter. Figure 12 shows this strategy parameterized with the percent completed and the delay time before a re-start. With this strategy, a suitable Lissajous orbit can be obtained but, in some instances, at the cost of a shadow (maximum depth of shadow is $4.1 \%$ ) in the cruise phase. The error incurred is again small (less than $4 \mathrm{~m} / \mathrm{s}$ ) and the $\Delta \mathrm{V}$ cost to remove the cruise shadow is small $(5 \mathrm{~m} / \mathrm{s})$ as well.

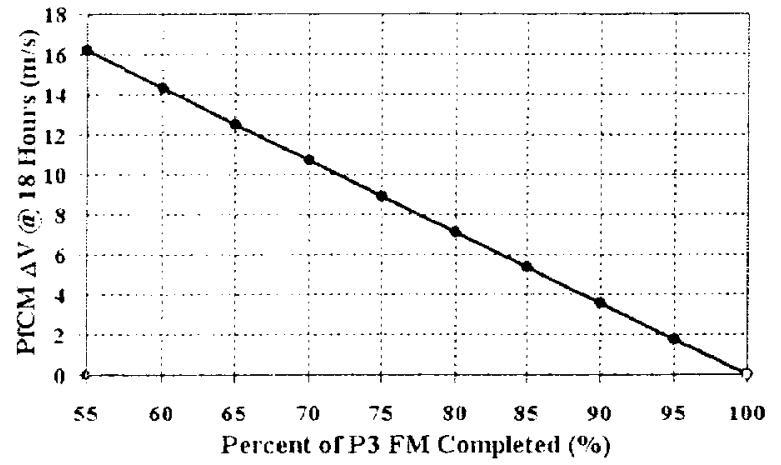

Figure 11: Penalty for Aborting P3 Maneuver

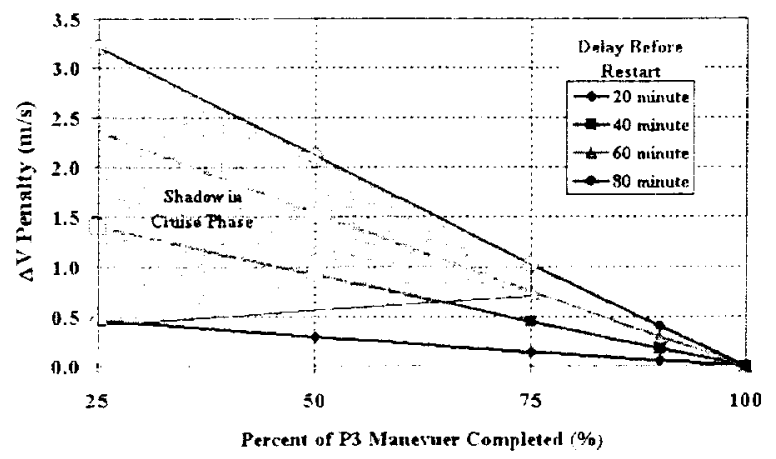

Figure 12: Penalty for Aborting | Restarting P3 Maneuver

\section{PfCM Maneuver}

As it was determined that PfCM was not a time-critical maneuver (a 12 hour delay only incurred a $17 \mathrm{~cm} / \mathrm{s}$ penalty), the abort scenarin for PfCM was not studied in any great detail.

\section{Missing a Maneuver}

The response strategy for missing a maneuver was the same as for the previous studies - opportunities at the downstream phasing loop maneuvers were used to compensate for the $\Delta \mathrm{V}$ loss. The penalties for missing a single maneuver are larger than in the previous contingency scenarios but still within the contingency allocation for the June 30 launch.

\section{PI Maneuver}

Despite the $\mathrm{Pl}$ maneuver being the largest maneuver scheduled during the phasing loops, the consequences of missing it are not the greatest. Absorbing the impact of missing P1 on the P2 and P3 maneuvers caused an overall $\Delta \mathrm{V}$ penalty of $10.6 \mathrm{~m} / \mathrm{s}$. In order to correct from missing the P1 maneuver, a P2 maneuver (not previously scheduled) of $34.2 \mathrm{~m} / \mathrm{s}$ would be needed while the P3 maneuver changed from a posigrade 8.7 $\mathrm{m} / \mathrm{s}$ to a retrograde $6.6 \mathrm{~m} / \mathrm{s}$. Essentially, the loss of the Pl maneuver forces great changes in the other maneuvers in order to meet the Moon at the appropriate time for the gravity assist.

\section{P2 Manetuer}

Unlike the P1 maneuver, there was a large penalty for missing the P2 maneuver - assuming the P1 maneuver was performed nominally. Missing the P2 maneuver $(2.5 \mathrm{~m} / \mathrm{s})$ required during operations would force the P3 maneuver to be moved significantly off of the perigee point (nearly 9 hours before perigee. This maneuver of $34 \mathrm{~m} / \mathrm{s}$ would slide backwards in order to compensate for the loss in the apparent apsidal line shift (as seen in the rotating frame) gained from the P2 maneuver. Furthermore, the location of this maneuver off of perigee would not allow the $+X$ thrusters to be used, as it would allow full Sun onto the instrument. In this case, the $+Z$ thrusters ( $3 \& 4$ ) would be needed in order to maintain thermal stability. A maneuver of this size using only the two $+Z$ thrusters (with their $30^{\circ}$ cant angles) would require over 100 minutes of thrusting. The P2 maneuver became a very crucial maneuver to perform. Regardless, the incurred penalty of $24 \mathrm{~m} / \mathrm{s}$ is within the June $30^{\text {th }}$ allocation.

\section{P3 Maneuver}

As the P3 maneuver is the last perigee maneuver prior to the lunar encounter, it was the last efficient place to add energy to the trajectory. Missing this $7.4 \mathrm{~m} / \mathrm{s}$ 
maneuver would have incurred a great cost at the PfCM planned for 18 hours after P3. In order to target back to a trajectory close to the nominal, $36.8 \mathrm{~m} / \mathrm{s}$ of $\Delta \mathrm{V}$ would be required. Such a maneuver is much greater than the $15 \mathrm{~m} / \mathrm{s}$ allocation for PfCM assumed before launch. Such a maneuver would require both tangential and normal components, with a yaw out of the orbit plane of $8.5^{\circ}$ to allow the normal component. The resultant trajectory satisfies the MAP trajectory constraints for Lissajous orbit size and zero shadows at $L 2$. Implementing this maneuver would be difficult as the $45^{\circ}$ limit on the Sun angle (with respect to the $+Z$ body axis) would be violated, allowing some Sun on the instrument. Fortunately, this did not occur but some important management decisions (e.g. whether or not to allow the Sun to shine on the instrument for the duration of the maneuver) would have been required to ensure mission success in this case.

\section{PfCM Maneuver}

Luckily, the predicted PfCM of $31 \mathrm{~cm} / \mathrm{s}$ was small due to the success of the three perigee maneuvers. This final small maneuver is still required due to the amplifying effects of the lunar encounter. Missing this small PfCM equated to performing a $13 \mathrm{~m} / \mathrm{s} \mathrm{MCC}$ maneuver seven days after the lunar encounter - a huge penalty. Fortunately, as was shown above, the PfCM isn't as time critical as the perigee maneuvers and even a twelve hour delay causes only a $17 \mathrm{~cm} / \mathrm{s}$ penalty. PfCM was performed nominally and required only a small $\mathrm{MCC}$ maneuver $(10 \mathrm{~cm} / \mathrm{s})$

\section{Missing the Lunar Encounter}

Analysis was performed to evaluate the capability of recovering from missing the final perigee maneuver and, hence, the lunar encounter ". This analysis was performed assuming all maneuvers had been successfully completed prior to the final perigee maneuver. If the lunar encounter has been missed, the spacecraft has to wait for about a full Moon revolution before being able to retarget the Moon, since desired Lissajous orbits result only when the lunar encounter occurs about 3 days before full Moon. A 30-day delay induces a line of apsides shift of about $30^{\circ}$ in the solar rotating frame. Thus, the Moon encounter has now shifted away from the desired Sun-Earth-Moon (SEM) angle. Indeed, in the rotating-frame, the line-of-apsides moves clockwise and the Moon moves counterclockwise, thus the SEM angle must decrease between the value of $125^{\circ}$ at the nominal encounter towards 90 degrees at the contingency encounter. Thus, the SEM angle is no longer favorable for achieving a desired Lissajous orbit. While the case studied was not the June 30, 2001 launch date, the size of the missed P3 maneuver $(6.9 \mathrm{~m} / \mathrm{s})$ was commensurate with the $\mathrm{P} 3$ maneuver from the June 30 launch date.

Two different strategies were studied to re-phase the spacecraft for the next lunar encounter. For Case 1, a maneuver is performed at the very next perigee after P3. Case 2 used three additional loops after the maneuver failure. In both cases, even though the spacecraft had "missed the Moon", its trajectory still got close enough to be changed by the close approach. Therefore, some $\Delta V$ was needed to correct the added energy from the Moon. For Case 1, the lunar encounter occurred about $90^{\circ}$ away from the Sun-Earth's line. Bending the trajectory to L2 required targeting for extremely high C3 at the Moon. In doing so, the spacecraft's energy was too high to be captured into a Lissajous orbit. Case 2 was implemented using three additional phasing loops. The idea was that the lunar encounter had to occur closer to the Sun-Earth's line to avoid the problem faced with Case 1. Indeed, a Lissajous orbit at L2 was achievable, as shown in Figure 13 , but at a $\Delta V$ expense of about $400 \mathrm{~m} / \mathrm{s}$. Table 2 presents the $\Delta V$ budget for the nominal and the two contingency trajectories. Both Case 1 and Case 2 require high energy maneuvers to encounter the Moon with peak maneuvers of $263 \mathrm{~m} / \mathrm{s}$ and $150 \mathrm{~m} / \mathrm{s}$ respectively. As stated earlier, because the SEM angle at lunar encounter is off the nominal value by about $30^{\circ}$, the resultant Lissajous orbit at L2 does not meet the MAP requirements of $0.5^{\circ}$ x $10.5^{\circ}$. More importantly, the $\Delta V$ required to implement this is beyond the capacity of MAP's propellant budget. As this was determined to be a catastrophic failure case, a great deal of work was expended in understanding the response scenarios for delaying, aborting, and/or restarting the P3 maneuver as a means to mitigate this risk.
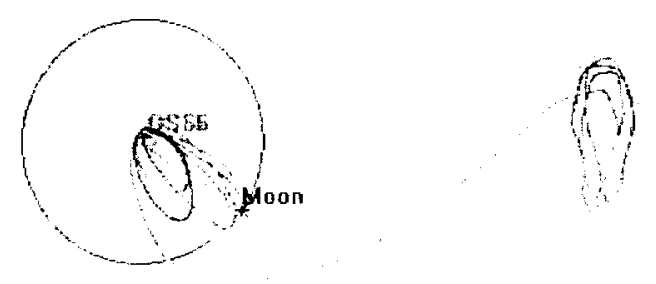

Figure 13: Recovery Attempt from Missing the Moon 
Table 2: Recovery $\Delta V$ for Missing the Moon

\begin{tabular}{|c|c|c|c|}
\hline Maneuver & Nominal & Case 1 & Case 2 \\
\hline $\mathrm{P} 1$ & 18.482 & 18.482 & 18.482 \\
\hline $\mathrm{P} 2$ & 0.000 & 0.000 & 0.000 \\
\hline $\mathrm{P} 3$ & 6.884 & 0.000 & 0.000 \\
\hline $\mathrm{P} 4$ & $\mathrm{n} / \mathrm{a}$ & 29.188 & 150 \\
\hline $\mathrm{A} 4$ & $\mathrm{n} / \mathrm{a}$ & 20 & 90 \\
\hline $\mathrm{P} 5$ & $\mathrm{n} / \mathrm{a}$ & 263.15 & $\mathrm{n} / \mathrm{a}$ \\
\hline $\mathrm{P} 6$ & $\mathrm{n} / \mathrm{a}$ & $\mathrm{n} / \mathrm{a}$ & 79.830 \\
\hline $\mathrm{P} 7$ & $\mathrm{n} / \mathrm{a}$ & $\mathrm{n} / \mathrm{a}$ & 54.846 \\
\hline Total & $\mathbf{2 5 . 3 6 6}$ & $\mathbf{3 3 0 . 8 2 0}$ & $\mathbf{3 9 3 . 1 5 8}$ \\
\hline
\end{tabular}

\section{Conclusion}

Several contingency studies for the MAP mission have been presented. Correcting for excessive launch vehicle errors up to $5 \sigma$ could cost up to $35 \mathrm{~m} / \mathrm{s}$. While the cost of splitting the first perigee maneuver to obtain DSN coverage from the ground was determined to be small $(<10 \mathrm{~m} / \mathrm{s})$, it was decided to execute maneuvers "in the blind" in the event of a TDRS outage. Analysis showed that delaying the perigee maneuvers was not too costly. Delaying the $\mathrm{P} 1$ maneuver cost around $10 \mathrm{~m} / \mathrm{s}$ while delaying $\mathrm{P} 2$ and $\mathrm{P} 3$ cost less than $6 \mathrm{~m} / \mathrm{s}$. It was discovered that adjusting the delayed P2 or P3 maneuver helped to reduce the penalty but in some cases lunar shadows re-appeared during the cruise phase. Aborting maneuvers after ignition induced penalties of less than $8 \mathrm{n} / \mathrm{s}$ for the perigee maneuvers but this penalty was minimized with an attempt to restart the aborted maneuver after a specified time (less than two hours). This ability to restart the maneuver would be highly dependent on the reason for the abort. Missing any of the perigee maneuvers was costly, though missing $\mathrm{P} 2$ or $\mathrm{P} 3$ resulted in large maneuvers (near $35 \mathrm{~m} / \mathrm{s}$ ) in order to recover. The recovery scenario for missing the P2 or P3 maneuvers resulted in maneuvering in non-optimal configurations (using two thrusters instead of four) or placing the instrument in partial view of the Sun, respectively. All of these contingencies could have been accommodated (singly) with the contingency $\triangle \mathrm{V}$ available for MAP's actual launch day $-47 \mathrm{~m} / \mathrm{s}$. Unfortunately, MAP would not have been able to recover from missing the Moon with the $\Delta \mathrm{V}$ available for maneuver design. As this was discovered, great effort was made in order to reduce the likelihood of this possibility. In the end, MAP did not suffer any contingencies that required extra maneuvering and MAP is now collecting data with the goal of producing a full-sky, high resolution map of the cosmic microwave background radiation some time in early 2003.

\section{References}

1. C. Brown, "Spacecraft Mission Design, Second Edition", American Institute of Aeronautics and Astronautics, Reston, VA, 1998, pp 115-117.

2. K. Richon and M. Matthews, "An Overview of The MAP Trajectory Design", AAS 97-728, August 1997.

3. O. Cuevas, L. Newman, M. Mesarch, and $M$. Woodard, "An Overview of Trajectory Design Operations for the Microwave Anisotropy Probe Mission", AIAA 2002-4425, Monterey, CA, August 2002.

4. G. Davis, "Trajectory Design \& Maneuver Operations Peer Review - RFA \#1 Response", MAP Project Document, December 2000.

5. M. Mesarch, D. Rohrbaugh, and C. Schiff, "Application Of Monte Carlo Analyses For The Microwave Anisotropy Probe (MAP) Mission", 16th International Symposium on Space Flight Dynamics, Pasadena, CA, December 2001.

6. C. Schiff, and L. Mailhe, "MAP Contingency Analysis: Launch Vehicle Dispersions Greater than $\pm 3 \sigma$ ", Memorandum to MAP Project, February 2001.

7. M. Mesarch, "Finite Maneuver Characteristics for the Microwave Anisotropy Probe (MAP) Mission", Memorandum to MAP Project, March 2001.

8. D. Rohrbaugh, "Splitting the First Perigee Maneuver", Memorandum to MAP Project, July 2000.

9. Loucks, M. and Helleckson, B., "Post-Swingby Targeting for MAP Lissajous Trajectories", Memo to MAP project, March 2000.

10. M. Mesarch and S. Andrews, "The Maneuver Planning Process for The Microwave Anisotropy Probe (MAP) Mission", AIAA 2002-4427, Monterey, CA, August 2002.

11. C. Schiff and L. Mailhe, "MAP Contingency Analysis: Missing the Moon", Memorandum to MAP Project, February 2001. 